\title{
Un análisis crítico de los debates acerca del duelo y el trastorno depresivo en la era del Manual Diagnóstico y Estadístico de los Trastornos Mentales
}

\author{
A critical analysis of debates on grief and depressive \\ disorder in the age of the Diagnostic and Statistical \\ Manual of Mental Disorders
}

Francisco Pizarro Obaid ${ }^{1}$, Rodrigo De La Fabián Albagli

${ }^{1}$ Psicólogo. Doctor en Sexualidad, Procreación y Perinatalidad. Profesor asociado, director de postgrado, Facultad de Psicología, Universidad Diego Portales, Santiago, Chile. $\triangle$ id

${ }^{2}$ Psicólogo. Doctor en Psicopatología Fundamental. Profesor asociado, Facultad de Psicología, Universidad Diego Portales, Santiago, Chile. $\triangle$ iD
RESUMEN Desde la incorporación del trastorno depresivo mayor en el Diagnostic and Statistical Manual of Mental Disorders (DSM-III) de 1980, hasta su actualización en el DSM-IV-TR, el sistema clasificatorio DSM consideró necesario incluir el criterio de "exclusión por duelo", con el objetivo de diferenciar la tristeza normal, vinculada a una pérdida, de un trastorno mental, como el trastorno depresivo mayor. En su última versión (DSM-5), esta excepción fue suprimida, dando lugar a una controversia que se extiende hasta nuestros días. El debate ha confrontado a quienes están a favor de mantener y extender la exclusión a otros estresores y aquellos que han querido erradicarla. Nuestra hipótesis es que estas posiciones darían cuenta de dos matrices clínicas y epistemológicas cualitativamente diversas ligadas a las trasformaciones mayores que han experimentado las ciencias de la salud y la psiquiatría. Mostramos que este debate involucró una renovación profunda del sentido de la práctica psiquiátrica, un cambio en la función del diagnóstico y el modo de concebir la etiología de la enfermedad mental, así como, una reformulación del estatuto del sufrimiento del paciente para el acto médico.

PALABRAS CLAVES Salud Mental; Trastorno Depresivo Mayor; Manual Diagnóstico y Estadístico de los Trastornos Mentales.

\begin{abstract}
Since the incorporation of the major depressive disorder in the Diagnostic and Statistical Manual of Mental Disorders (DSM-III) in 1980, and until its update in the DSMIV-TR, the DSM classification system considered it necessary to include the criterion of "bereavement exclusion", with the aim of differentiating normal sadness linked to a loss, from a mental disorder, such as the major depressive disorder. In its latest version (DSM5), this exception was removed, giving rise to a controversy that continues to this day. The debate has set those who are in favor of maintaining this exclusion and extending it to other stressors against those who have intended to eradicate it. Our hypothesis is that these positions account for two qualitatively diverse clinical and epistemological matrices, linked to major transformations in health sciences and in psychiatry. We show that this debate involved a profound renewal of the meaning of psychiatric practice, a change in the function of diagnosis and in the way of conceiving the etiology of mental disorders, as well as a reformulation of the patient's suffering status for the medical act.
\end{abstract}

KEY WORDS Mental Health; Major Depressive Disorder; Diagnostic and Statistical Manual of Mental Disorders. 


\section{INTRODUCCIÓN}

Hacia fines de la década de 1970, la depresión no era más que un síntoma detectable en la mayor parte de las enfermedades mentales y no constituía un objeto relevante de atención en nuestras sociedades ${ }^{(1)}$. Sin embargo, desde principios de la década de 1980, se registró un aumento exponencial en su diagnóstico y el trastorno depresivo se convirtió en una de las condiciones psiquiátricas más frecuentes $\mathrm{s}^{(2,3,4,5)}$.

El incremento sostenido del diagnóstico ha llevado a la Organización Mundial de la Salud $^{(6)}$ a estimar que, entre 2005 y 2015 las personas que vivían con depresión en el mundo aumentaron un $18,5 \%$, llegando, en 2017, a un total de afectados que excedería los 300 millones. Esta cifra representa un $4,4 \%$ de la población mundial siendo, además, la patología que mundialmente más contribuye a los años de vida con discapacidad. Se calcula que en los próximos años, alrededor de dos tercios de la población mundial cumplirá con los criterios diagnósticos del trastorno depresivo mayor en algún momento de la vida ${ }^{(5)}$. El aumento del diagnóstico de depresión ha estado vinculado, asimismo, al significativo incremento del uso de antidepresivos ${ }^{(7,8)}$, constatándose, por ejemplo, que en los países pertenecientes a la Organization for Economic Co-operation and Development (OECD) $)^{(9)}$ el consumo de estos psicofármacos se ha duplicado en el período comprendido entre 2000 y 2015.

La prevalencia del trastorno depresivo y el alarmante incremento en el uso psicofármacos ha llevado a considerar a la depresión como una crisis global para la salud pública(10) y una verdadera "epidemia moderna"(11).

Wakefield y Demazeux ${ }^{(12)}$ señalan que en la amplia literatura que intenta explicar las causas del aumento explosivo de esta patología habría, fundamentalmente, dos tipos de hipótesis. La primera, denominada perspectiva realista, supone que el fenómeno tendría causas "reales" u "objetivas", tales como el exceso de individualismo imperante en las sociedades neoliberales o desbalances neuroquímicos mejor diagnosticados, entre otros. La segunda, en cambio, considera el boom de la depresión como efecto de una inflación artificial del diagnóstico, ligada, principalmente, a la publicación de la tercera versión del Manual Diagnóstico y Estadístico de los Trastornos Mentales o DSM-III(2). donde se incluyó, por primera vez, la categoría de "trastorno depresivo mayor"(3).

El presente artículo tiene por objetivo analizar una de las dimensiones más actuales y relevantes del debate entre representantes de estos dos modos de entender el auge contemporáneo de la depresión: la controversia provocada, a propósito de la publicación de la quinta y última versión del manual de psiquiatría DSM-5 ${ }^{(13)}$, por la eliminación del duelo como un criterio diagnóstico diferencial, en el campo de los trastornos del ánimo ${ }^{(14,15,16)}$. Para Ronald Pies

Sin lugar a dudas, esta fue una de las decisiones más polémicas que realizó el grupo de trabajo del DSM-5; y, de alguna manera, la decisión más controvertida de la American Psychiatric Association (APA) desde que se eliminó la homosexualidad de la lista de trastornos psiquiátricos en 1973 [Without question, this was one of the most contentious decisions the DSM-5 work groups made - and, by some lights, the most controversial decision by the American Psychiatric Association (APA) since homosexuality was removed from the list of psychiatric disorders in 1973] ${ }^{(17)}$.

En efecto, desde el DSM-III ${ }^{(2)}$, y a lo largo de sus actualizaciones -DSM-III-R ${ }^{(18)}$, DSM-IV ${ }^{(19)}$; DSM-IV-TR ${ }^{(20)-}$, el manual consideraba necesario distinguir el trastorno depresivo mayor del duelo. De este modo, la "exclusión por duelo" (bereavement exclusión) suponía que, si luego de la muerte de un ser querido, se presentaba un cuadro sintomáticamente equivalente a un trastorno depresivo mayor, hasta de dos meses de duración -no agravada por síntomas tales como ideación suicida o psicóticos, por ejemplo-, se lo debía comprender como una reacción normal ante la pérdida. 
Sin embargo, a partir de la publicación del DSM-5 en $2013^{(13)}$ este criterio diferencial fue eliminado bajo la primacía del síntoma, descartando el valor de cualquier circunstancia biográfica concomitante a la enfermedad.

Mientras los especialistas que abogaron a favor de suprimir el criterio de exclusión por duelo del DSM-5, inspirados por una perspectiva realista acerca del trastorno depresivo mayor, buscaban aminorar la posibilidad de generar falsos negativos $y$, consecuentemente, disminuir el riesgo del subdiagnóstico de esta patología ${ }^{(21,22,23,24,25)}$, otros autores consideraban que la exclusión por duelo no solo era imprescindible, sino que debía extenderse a nuevos estresores (pérdida del trabajo, rompimiento de vínculos afectivos, etc.), ya que su eliminación conduciría, irrevocablemente, a producir falsos positivos y, con ello, a un sobrediagnóstico artificial de la patología ${ }^{(26,27,28,29,30,31)}$.

A partir de estos antecedentes, el presente artículo supone que la comprensión de los debates entre trastorno depresivo y duelo, derivados de las innovaciones del DSM-5, requiere tanto de un análisis de la evolución de los criterios relativos a los trastornos del ánimo en el sistema clasificatorio DSM, así como la consideración de las transformaciones que experimentó la matriz clínica y epistemológica de las ciencias de la salud, en general, y de la psiquiatría en particular. La relevancia de esta investigación teórica radica, en primer lugar, en el desearrollo de un análisis crítico original acerca de la evolución del que probablemente sea el manual de diagnóstico psiquiátrico más influyente en Latinoamérica y el mundo:

[su influencia se extiende] por todas las regiones y los países del mundo, convirtiéndolo no solo en el best seller más exitoso de la literatura psiquiátrica sino en punto de referencia obligado en toda labor clínica, docente, administrativa, legal o heurística a nivel universal. ${ }^{(32)}$

En segundo lugar, propone, desde la noción de "salud colectiva"(33,34), un análisis sociohistórico y genealógico del proceso de construcción de una categoría diagnóstica dominante ${ }^{(35)}$, como lo es el trastorno depresivo mayor. Bajo estas coordenadas se sostendrá que la comprensión de los problemas involucrados en el debate acerca de la eliminación de la exclusión por duelo, requiere la consideración de las transformaciones que ha registrado el modelo epistemológico hegemónico en las ciencias biomédicas, ya que estas modificaciones han implicado una renovación profunda del sentido y objetivo de la práctica psiquiátrica, una variación en la función del diagnóstico, innovaciones en el modo de concebir la etiología de la enfermedad mental, así como también, cambios en el estatuto del sufrimiento del paciente para el acto médico.

\section{ANTECEDENTES}

\section{El debate en torno al duelo, la tristeza y la depresión: la evolución histórico- conceptual del DSM}

Las periódicas actualizaciones de la clasificación del DSM y su pragmática implementación en el campo de la salud mental hacen olvidar, a menudo, las notables diferencias que es posible apreciar entre los fundamentos y criterios que dieron forma a sus versiones iniciales y los principios que sustentaron la elaboración de sus últimos manuales. En este sentido, el paso paradigmático del DSMII al DSM-III, fue decisivo para la reconfiguración de los trastornos del ánimo, y el cambio desde la versión IV a la $\mathrm{V}$ tuvo una incidencia crítica en los destinos del duelo y la depresión.

Al establecer una revisión histórico-conceptual de las transformaciones del DSM, es posible constatar que las dos primeras versiones del manual estuvieron fuertemente influenciadas por las concepciones etiológicas de la teoría psicoanalítica, por la perspectiva psicobiológica de Adolph Meyer, así como por la teoría de Karl Meninger que, bajo una perspectiva psicosocial, consideraba a la enfermedad mental como una dificultad de adaptación del individuo a su entorno ${ }^{(36,37)}$. 
En términos generales, según Wilson ${ }^{(38)}$, estos dos manuales compartían algunos puntos centrales: consideraban que había una continuidad dimensional entre lo normal y lo patológico ${ }^{(39)}$, diferenciaban nítidamente los trastornos psicóticos de los trastornos neuróticos y, por último, compartían un cierto descrédito por el modelo diagnóstico descriptivo de Kraepelin, en favor del modelo etiológico intrapsíquico del psicoanálisis.

Las consecuencias de estos supuestos tuvieron directa influencia en la comprensión de los trastornos del ánimo, ya que ambos manuales consideraban pertinente discriminar si lo depresivo se registraba en el ámbito de los trastornos psicóticos o en el de los psiconeuróticos ${ }^{(40)}$. El DSM-I ${ }^{(41)}$, en concordancia con Mayer, utilizaba la categoría de "reacción" psicopatológica, diferenciando las "reacciones psicóticas" -en sus dos modalidades, maniacodepresiva y depresiva- de la "reacción depresiva", la cual formaba parte de las psiconeurosis. Por su parte, el DSM-II ${ }^{(42)}$ decidió eliminar la noción de "reacción" y extendió la influencia del psicoanálisis, distinguiendo entre trastornos afectivos mayores o psicosis afectivas y neurosis depresiva.

Pero los cambios en los criterios y en los supuestos referidos a la enfermedad mental que registraron las primeras versiones del manual no se limitaron a un problema puramente nosográfico, sino que provocaron cambios en la comprensión de lo normal y lo patológico, tanto a nivel médico, como social. En términos generales, promovieron un proceso gradual de ampliación de los límites de la psiquiatría más allá de la población institucionalizada y extendieron sus competencias hacia la vida cotidiana de las personas, reconociendo la influencia de factores ambientales en el desencadenamiento de psicopatología de gravedad moderada, como las psiconeurosis. Según Grob, el DSM-I y el DSM-II conllevaron

...una ampliación extraordinaria de los límites psiquiátricos y un rechazo de la tradicional distinción entre salud mental y anormalidad mental. Pasar de una preocupación por las enfermedades mentales en poblaciones institucionales a la incidencia en la población general representó un salto intelectual extraordinario [an extraordinary broadening of psychiatric boundaries and a rejection of the traditional distinction between mental health and mental abnormality. To move from a concern with mental illnesses in institutional populations to the incidence in the general population represented an extraordinary intellectual leap]. ${ }^{(43)}$

Si el paso a su segunda versión fue significativo, la publicación de su tercera versión marcó un hito cualitativo, al distanciarse definitivamente de los modelos etiológicos psicógenos o los principios de la psiquiatría tradicional, ya que

...el DSM-III transformó radicalmente la naturaleza de la enfermedad mental. En un notable breve periodo de tiempo, la psiquiatría se deshizo de un paradigma intelectual y adoptó un sistema clasificatorio completamente nuevo [DSM-III radically transformed the nature of mental illness. In a remarkably short time, psychiatry shed one intellectual paradigm and adopted an entirely new system of classification]. ${ }^{(44)}$

Los factores que influyeron en esta transformación son heterogéneos. En primer lugar, durante las décadas de 1960 y 1970, la crítica antipsiquiátrica al diagnóstico y la legitimidad de la profesión se ligó a un esfuerzo por validar, científica y políticamente, los criterios diagnósticos ${ }^{(36)}$. Asimismo, desde la década de 1970 emergió un descontento con el modelo psicoanalítico y un creciente interés en la perspectiva diagnóstica descriptiva de corte neo-kraepeliana ${ }^{(38)}$. En tercer lugar, la poca claridad en la definición de lo normal y lo patológico, desperfilaba el rol del médico, el cual debía empezar a competir con una serie de otros profesionales -psicólogos, consejeros, etc.- en el tratamiento de patologías menos severas. Por último, el desarrollo de nuevas formas de medición estandarizadas -escalas de ansiedad o de depresión, por 
ejemplo- así como el desarrollo de la psicofarmacología y su influencia decisiva en los modos de producir y distinguir las categorías diagnósticas ${ }^{(40)}$ generó, junto a los demás cambios mencionados, las condiciones propicias para la revolución en la psiquiatría que implicó la publicación del DSM-III.

El giro paradigmático que registró el sistema clasificatorio implicó, en términos generales, el rechazo de la división fundamental entre neurosis y psicosis -con lo que se daba vuelta a la página a más de un siglo de psiquiatría-, así como la farmacologización y, por ende, la re-medicalización de la psiquiatría, para reducir el diagnóstico a un signo descriptivo definido bajo parámetros temporales (duración, frecuencia) y criterios de intensidad (leve, moderado, agudo). Más específicamente, en lo relativo a la incorporación del trastorno depresivo mayor, este quedó enteramente contenido bajo la categoría de trastornos del ánimo, dentro de los cuales se distingue el trastorno depresivo mayor, entendido como una patología episódica que puede o no tener síntomas psicóticos, y la distimia, entendida como una patología leve y crónica. Asimismo, con el objetivo de limitar la acción psiquiátrica y aminorar la posibilidad de confundir una reacción depresiva normal con un trastorno ${ }^{(15)}$, se instauró la exclusión diagnóstica por duelo normal.

Los antecedentes de la exclusión por duelo o bereavement exclusion como se conoce en inglés, se remontan a más de un siglo $^{(45)}$. Sin embargo, dentro de ellos se destacan dos: en primer lugar, la distinción entre duelo normal y patológico propuesta por Sigmund Freud, en 1917, en su clásico texto Duelo y melancolía ${ }^{(46)}$; y, en segundo lugar, una investigación realizada por Clayton, Desmarais y Winokur en $1968^{(47)}$, en la cual se evidencia que, en el proceso de duelo, una minoría significativa tiende a desarrollar un número considerable de síntomas depresivos que remiten espontáneamente dentro de las seis a diez semanas posteriores a la pérdida. Para poder establecer el diagnóstico diferencial, tanto para el DSM III, como para el DSM-III-R, resultaba necesario especificar el estatuto del duelo y su evolución. Al duelo normal lo llamaron "duelo no complicado" y lo distinguieron, por una parte, del duelo complicado, caracterizado por la aparición de síntomas depresivos severos o por síntomas menos severos prolongados en el tiempo tras la pérdida y, por la otra, del trastorno depresivo mayor, propiamente tal, el cual no se diagnosticaba en concomitancia con la pérdida de un ser querido, salvo si cursaba con un duelo complicado. Ahora bien, este criterio de excepción por duelo se mantuvo prácticamente inalterado hasta la cuarta versión revisada del DSM (DSM-IV-TR), siendo eliminada en el DSM-5.

La exclusión del duelo normal de la última versión del manual transcurre en el contexto de un socavamiento de la diferencia entre lo normal y lo patológico. Tres años antes de la publicación del DSM-5, Allen Frances advertía sobre este peligro:

EI DSM-5 parece estar promoviendo lo que más hemos temido: la inclusión de muchas variantes normales en la rúbrica de la enfermedad mental, con el resultado de que el concepto central de "trastorno mental" se ve socavado en gran medida [DSM5 appears to be promoting what we have most feared - the inclusion of many normal variants under the rubric of mental illness, with the result that the core concept of "mental disorder" is greatly undermined]. ${ }^{(48)}$

Tal como lo señala Joel Paris ${ }^{(49)}$, una de las características de este manual es hacer cada vez más tenue, y menos relevante para el quehacer médico, la diferencia entre lo normal y lo patológico.

Algunas de las transformaciones estructurales más importantes introducidas en el DSM-5 y que apuntan en este sentido son: en primer lugar, la eliminación del sistema multiaxial de diagnóstico ${ }^{(50)}$ que, desde el DSM-III, había configurado al manual en torno a cinco ejes. Este cambio se tradujo en la desaparición del último resabio de distinción entre neurosis y psicosis, es decir, entre patologías propiamente psiquiátricas y patologías psicoterapéuticas que, de alguna 
manera, era conservada por los ejes I (síndromes clínicos o trastornos mentales) y II (retraso mental y trastornos de personalidad). Asimismo, la eliminación del eje IV dejó sin registro a los factores psicosociales y contextuales, es decir, los antiguos estresores o desencadenantes de las patologías mentales ${ }^{(39)}$. Por último, el DSM-5 realizó una crítica al diagnóstico categorial que se impuso desde el DSM-III, reconociendo, oficialmente, el carácter continuo y dimensional de los trastornos mentales, pero ya no basado en la teoría psicoanalítica -como en el caso del DSM-I y II-, sino en el modelo dimensional de las neurociencias ${ }^{(39)}$.

En su conjunto, se puede afirmar que la evolución de casi 60 años de este manual diagnóstico pone en evidencia, no solo cambios nosográficos, sino un proceso de transformación mayor de las ciencias de la salud, en general, y de la psiquiatría en particular. A partir de la década de 1950, la caída de la distinción entre patologías psiquiátricas, propias de pacientes institucionalizados, y patologías menos severas, propias de la vida cotidiana, propició una transformación de las tecnologías de la salud en tecnologías de gestión de la vida, puesto que ahora

...las tecnologías médicas contemporá-
neas no solo buscan curar enfermeda-
des, sino controlar y gestionar procesos
vitales del cuerpo y la mente. Ya no son
más tecnologías de la salud, sino tec-
nologías de la vida. [...contemporary
medical technologies do not merely
seek to cure diseases, but to control and
manage vital processes of the body and
mind. They are no longer technologies
of health but technologies of life]. ${ }^{(51)}$

Ahora bien, aun cuando el DSM-III buscó definir con mayor claridad los límites entre lo normal y lo patológico, así como la frontera entre el campo de acción propiamente psiquiátrico y otras prácticas no médicas, el resultado parece haber sido el inverso. En efecto, el rechazo de la división fundamental entre neurosis y psicosis y la redefinición descriptiva y ateórica del diagnóstico solo contribuyó a desdibujar aún más dichos límites y a expandir el campo de acción de la psiquiatría hacia la vida cotidiana de las personas. De este modo, según Rose, en la década de 1980, la psiquiatría sufrió una significativa transformación:

...se había convertido en algo mucho
más que una especialidad para el manejo
de una pequeña minoría de personas
que no podían vivir en el mundo del tra-
bajo, la familia y la civilidad, se había
convertido en una "disciplina de la salud
mental" generalizada, cuya racionali-
dad no era tanto la cura como el "hacer
frente", ayudando a las personas con
problemas a gestionar sus vidas cotidia-
nas. [...had become much more than a
specialty for the management of a small
minority of persons unable to live in the
world of work, family, and civility - it
had become a widespread "discipline of
mental health" whose rationale was not
so much cure as "coping" helping trou-
bled individuals manage themselves in
their everyday lives].

Este proceso de expansión de la psiquiatría hacia la vida cotidiana de las personas, que implicó la redefinición de los clásicos criterios de salud y enfermedad, de lo normal y lo patológico, se vio acentuada con la publicación del DSM-5 en el año 2013. Si el modelo médico tradicional proponía una práctica terapéutica situada en el eje normal-patológico, las nuevas tecnologías parecen no distinguir entre una acción que busca reestablecer la salud, de otra que se orienta a mejorar la vida de las personas, situándose en un eje distinto: sufrimiento-bienestar. Bajo estas consideraciones, la psiquiatría del siglo $\mathrm{XXI}$ se va transformando en una tecnología que ya no tiene por objetivo principal "curar" la enfermedad, sino que busca la "maximización del potencial individual, la minimización de la tristeza y la ansiedad, la promoción del bienestar, incluso de la felicidad [maximization of individual potential, the minimization of sadness and anxiety, the promotion of wellbeing, even happiness]"(52). 
Es en este contexto que el debate acerca del duelo y la depresión adquiere su relevancia y su épica. Más que una psicopatología en particular, la distinción interpela la labor médica y confronta al saber psiquiátrico con dos maneras muy heterogéneas de entender el quehacer clínico.

\section{ANÁLISIS CRÍTICO DEL DEBATE}

\section{La exclusión del duelo normal del diagnóstico de depresión: de la normalización a la promesa de bienestar}

Hacia fines de la primera década del siglo XXI, la preparación del DSM-5 dio inicio a una intensa discusión respecto a la pertinencia de mantener o eliminar la distinción entre duelo normal y trastorno depresivo. Este apasionante debate, que está lejos de agotarse, se desarrolló en el ámbito académico, en columnas de opinión, programas radiales y entrevistas realizadas a los principales involucrados en él y fue exhaustivamente recopilado ${ }^{(14,15,53)}$. Por lo tanto, en términos metodológicos, centraremos el análisis en los textos, tanto científicos como de divulgación científica, que temporalmente se sitúan en los años inmediatamente previos a la publicación del DSM-5. La razón es que en ellos asistimos al proceso de cristalización de las dos posiciones muy claramente diferenciadas, las cuales animan el debate, sin transformaciones sustantivas, hasta el día de hoy. Al respecto, nuestra hipótesis es que, examinadas en perspectiva, las diferencias entre estas dos posiciones no se redujeron a una cuestión meramente clasificatoria o factual. Prueba de esto, tal como lo veremos, es que los resultados de las investigaciones no se distinguieron significativamente, sino que el peso recayó, más bien, en los modos heterogéneos de interpretarlos y en las consecuencias clínicas derivadas de una u otra posición.

La publicación de The loss of sadness ${ }^{(27)}$ (La pérdida de la tristeza), en 2007, y las posteriores investigaciones de Jerome $\mathrm{C}$. Wakefield y sus colaboradores marcaron una clara posición frente al problema. Desde su perspectiva, la definición de un diagnóstico estrictamente descriptivo de la depresión, es decir, sin consideración alguna por el contexto, tuvo por consecuencia la confusión entre tristeza normal y patológica, produciendo un aumento explosivo de falsos positivos. Esta tesis se vio reforzada por la investigación que realizaron ese mismo año ${ }^{(26)}$, al comparar episodios con síntomas depresivos no complicados, vinculados a la pérdida de un ser querido, con episodios similares vinculados a otros tipos de pérdidas, como la del vínculo marital o la pérdida inesperada del trabajo, entre otras. La conclusión a la cual llegan estos autores es que no existía una diferencia significativa entre estos dos grupos, postulando que la exclusión por duelo debería ampliarse a otros tipos de estresores, con el fin de restringir el diagnóstico de trastorno depresivo mayor.

En contraposición a Wakefield et al., Zisook, Shear y Kendler ${ }^{(21)}$ emprendieron una revisión de las investigaciones en las cuales se comparaban episodios depresivos vinculados al duelo -tanto episodios complicados, como no complicados- con toda otra clase de episodios depresivos, concluyendo que no existían diferencias significativas entre estos dos grupos. Un año más tarde estos hallazgos fueron ampliados por Kendler, Myers y Zisook ${ }^{(22)}$ poniendo en evidencia que no habría discrepancias significativas entre episodios depresivos -complicados y no complicados- vinculados al duelo, con episodios depresivos -complicados y no complicados-, vinculados a otro tipo de estresores. Ahora bien, pese a las similitudes que podría haber con los resultados de Wakefield et al. ${ }^{(26,27)}$, Kendler et al. ${ }^{(21,22)}$ Ilegaron a una interpretación opuesta. A partir de ella, refutaron la validez de la exclusión por duelo para el diagnóstico de depresión mayor y advirtieron que "Extender esta exclusión a otros eventos de pérdida podría crear un desastre de salud pública. Nuestros pacientes merecen algo mejor [Extending this exclusion to still other loss events could create a public health disaster. Our patients deserve better]"(54). 
De este modo, resulta claro que esta controversia no se reduce a una discusión meramente factual sobre hallazgos científicos heterogéneos. Justamente, para poder comprender lo que está en juego es necesario realizar un análisis crítico del modo en que estas posiciones entienden aspectos centrales de la práctica clínica y de los trastornos mentales.

Bajo esta perspectiva, la posición de Wakefield y Horwitz implica que la tarea del profesional de la salud no es solo, ni primordialmente, tratar de aliviar el sufrimiento, ya que antes de proponérselo, el clínico debería formular y responder una pregunta prioritaria: ¿es o no, tal o cual sufrimiento, normal o patológico?

Si bien es cierto que los médicos siempre han ayudado a las personas con sufrimiento -con o sin un trastorno- también han abordado explícitamente la cuestión diagnóstica de si la condición de aflicción del paciente es normal o patológica. [...] La distinción entre la tristeza normal y patológica es igualmente real a pesar de la borrosidad considerable de los límites, con casos claros en ambos lados, algunos de los cuales están mal clasificados según los criterios del DSM. [While it is true that physicians have always helped suffering people - with or without a disorder - they have also explicitly addressed the diagnostic issue of whether the patient's distressed condition is normal or disordered. [...] The distinction between normal and disordered sadness is similarly real despite considerable boundary fuzziness, with clear cases on both sides - some of which are misclassified by DSM criteria]. ${ }^{(55)}$

Para abordar este desafío, Wakefield ${ }^{(53)}$ propone un tipo de análisis diagnóstico de los trastornos mentales Ilamado "disfunción dañina" (harmful dysfunction). Este procedimiento supone que para que una condición psiquiátrica sea catalogada como trastorno, debe ser juzgada como "dañina", de acuerdo con los valores sociales y evaluada como "disfuncional", con relación al supuesto "diseño biológico" de un mecanismo físico o psicológico. Es decir, el primero de ellos asocia la distinción entre lo normal y lo patológico, a lo que una sociedad tradicionalmente considera al respecto; mientras que el segundo, compara el funcionamiento de un individuo con el diseño que la evolución de las especies le ha otorgado a sus distintos mecanismos psicológicos y biológicos.

Por lo tanto, recurriendo a lo socialmente admitido y la función evolutiva de la tristeza, Wakefield et al. conciben la "reacción depresiva no complicada" como normal, en tanto respuesta adaptativa y proporcional ante una pérdida. Así, la función primordial del saber médico sería, a partir de ciertos criterios normativos externos al sufrimiento del paciente, determinar el límite entre lo normal y patológico, con el fin de enmarcar su praxis en esta dicotomía y evitar el peligro de "medicalizar" la vida normal.

En contraposición a esta perspectiva, los partidarios de suprimir la exclusión por duelo definen este modo de diagnosticar como una "falacia de la empatía mal situada"(25). Esta falacia se caracteriza porque un profesional bien intencionado puede, paradójicamente, fallar el diagnóstico de trastorno depresivo mayor por creer "comprender" que "cualquier persona" que se enfrenta a un estresor grave debería sentirse deprimido. Acentuando su crítica, subrayan que

\footnotetext{
...simplemente no se sigue lógicamente que, solo porque la reacción de alguien ante un evento sea "comprensible", no pueda ser patológica y, en muchos casos, severamente debilitante [...it simply does not follow logically that, just because one's reaction to an event is "understandable", it cannot be pathological and in many cases severely debilitating]. ${ }^{(25)}$
}

Asimismo, añaden, la principal tarea del médico no sería distinguir si un sufrimiento es o no normal, es decir, proporcional o no respecto de un estresor, sino la de intentar aliviarlo.

Pies $^{(23)}$ ilustra esta crítica mediante una analogía muy elocuente: el médico enfrentado al desafío de diagnosticar un infarto 
al miocardio no se pregunta si fue causado por malos hábitos alimentarios o si irrumpe de manera espontánea e incomprensible. Al momento de analizar el síntoma, en nada le importa el contexto, ya que siempre se lo considera dañino y se lo trata como tal. Por lo mismo, criticando directamente el enfoque evolucionista de Horwitz y Wakefield, Pies concluye:

...la función principal del médico siempre ha sido aliviar el sufrimiento y la incapacidad, no actuar como un biólogo evolutivo aficionado y juzgar desde un sitial elevado, qué tan "proporcional" es la respuesta de un paciente a algún estresante putativo. [...the physician's primary role has always been to relieve suffering and incapacity - not to act as an amateur evolutionary biologist and sit in lofty judgment, as regards how "proportionate" a patient's response is to some putative stressor]. ${ }^{(23)}$

Por lo tanto, los debates sobre la consideración o no del duelo como una entidad distinta del trastorno depresivo mayor, ilustran dos modos heterogéneos de comprender el sentido o telos de la práctica médica. En un caso, resulta prioritaria la distinción entre lo normal y lo patológico, tarea que supone clasificar el sufrimiento del paciente desde criterios normativos externos; en el otro, el sentido fundamental de la práctica médica es la de aliviar el sufrimiento, por lo cual, determinar si es o no normal ya no es un asunto de vital importancia. De este modo, mientras Wakefield et al. comprenden la práctica médica en el eje normal-patológico, Kendler y colaboradores la entienden al servicio de la calidad de vida de los pacientes, es decir, en el eje sufrimiento-bienestar.

Pero las diferencias que sostienen los protagonistas del debate no se agotan en las consideraciones relativas a las posibles relaciones entre los normal y lo patológico. Las diferentes posiciones sobre el problema se traducen, a su vez, en distintos modos entender la etiología de la depresión y justificar su diagnóstico.
Pies argumenta que la noción de "desencadenante" de una enfermedad involucra problemas clínicos y epistemológicos considerables. Desde su perspectiva no es clínicamente relevante saber si una enfermedad fue o no gatillada por algún estresor, además de ser prácticamente imposible definirlo, pues hay distorsiones de distinta índole -cronológicas, médicas, entre otras- que hacen poco fiable la información. Si hubiese un desencadenante médicamente relevante debería ser de orden neuromolecular:

...el constructo de un "desencadenante" depresivo es nebuloso y empíricamente inverificable, excepto quizás en escenarios altamente inusuales (por ejemplo, se le inyecta a un sujeto eutímico un potente agente biogénico de corta duración que disminuye la amina; síntomas depresivos se desarrollan dentro de las dos horas siguientes y espontáneamente remiten dentro de las próximas doce horas). [... the construct of a depressive 'trigger' is nebulous and empirically unverifiable, except perhaps in highly unusual scenarios (for instance, a euthymic subject is injected with a powerful, short-acting biogenic amine-depleting agent; severe depressive symptoms develop within 2 hours and then spontaneously remit over the next 12 hours)]. ${ }^{(23)}$

Por lo mismo, continúa Pies, la única forma para determinar si efectivamente el diagnóstico de trastorno depresivo mayor dentro de los dos meses siguientes a una pérdida es un falso positivo, sería contar con un "test para la depresión" que, a nivel neuro-molecular, pudiese dar cuenta de una respuesta cierta. De este modo, la depresión ha devenido un hecho molecular, pues es a esta escala en la que se puede justificar la verdad o falsedad de un diagnóstico.

Para Wakefield et al., la dimensión neuromolecular del trastorno depresivo mayor no permite, por sí misma, determinar si un diagnóstico es verdadero o falso ${ }^{(6)}$. Incluso si un eventual "test de depresión" estableciera que podría haber un trastorno depresivo mayor, 
este dato no zanjaría la cuestión de si se trata de una patología o de una reacción normal. La depresión como patología sería un constructo "hibrido"(56), con bases biológicas, pero que no puede existir, como tal, sino es en un contexto social y evolutivo que la distinga de la tristeza normal.

Por lo tanto, ambas posiciones coinciden en que el sufrimiento de un paciente no se vincula directamente con la verdad del diagnóstico, puesto que, en un caso, la verdad es contextual y, en el otro, neuromolecular. Sin embargo, ellas difieren en el estatuto que tiene el sufrimiento en sus respectivas clínicas, más específicamente, en el vínculo entre el sufrimiento y los modos de dar legitimidad al acto médico. Para Wakefield et al., el padecer o el alivio no basta para justificar, ni comprender el acto médico. Por ejemplo, aun cuando un medicamento puede producir efectos benéficos en personas con o sin una patología de base, para estos autores resulta fundamental discriminar entre estas dos situaciones, por lo que sostendrán:

No nos oponemos a la medicación de pacientes con sufrimiento normal; más bien, nos oponemos a etiquetar erróneamente las afecciones como trastornos, lo que sesga el pronóstico, el consentimiento informado y la planificación del tratamiento. [We are not opposed to medicating patients with normal distress; rather, we object to mislabeling conditions as disorders, thus biasing prognosis, informed consent, and treatment planning]. ${ }^{(5)}$

Es decir, el acto de medicar no encuentra su legitimidad en el sufrimiento del paciente, sino en la distinción normal/patológico, de modo de no confundir la terapéutica, con la búsqueda del bienestar.

Por otra parte, para Kendler et al., en la medida en que ellos rechazan situarse en una exterioridad normativa desde donde evaluar la "proporcionalidad" del sufrimiento, aumentar el bienestar del paciente deviene tanto el objetivo clínico fundamental, como la razón por la cual se realiza el acto médico.
Tal como señala Rose ${ }^{(57)}$, este modo particular de comprender la clínica se vincula con transformaciones mayores en las ciencias biomédicas ocurridas en los últimos años, las cuales se caracterizan por haber tornado indistinguible la corrección terapéutica, del potenciamiento y la búsqueda del bienes$\operatorname{tar}^{(58)}$. Es decir, para que un acto médico sea legítimo, ya no es urgente determinar la verdad del diagnóstico, sino el grado de alivio que podría aportarle al paciente, el cual, a su vez, deviene un nuevo paciente-consumidor, que no espera ser normalizado, sino recibir una respuesta a sus demandas de mayor bienestar.

En términos generales, se puede afirmar que las posiciones analizadas expresan dos matrices clínicas y epistemológicas diversas, puesto que ellas divergen en aspectos decisivos, tales como: el sentido del quehacer psiquiátrico, la función del diagnóstico, la etiología de la enfermedad mental, así como el estatuto del sufrimiento del paciente para el acto médico.

\section{CONCLUSIÓN}

Al examinar críticamente los debates sobre la exclusión del duelo en el DSM-5 resulta claro que el problema rebasa los argumentos meramente clasificatorios. Por una parte, da cuenta de ciertas transformaciones radicales en la racionalidad, las tecnologías y el imaginario de las ciencias biomédicas de los últimos años $y$, por otra parte, ilustra el paso de una práctica psiquiátrica entendida como tecnología de normalización, a una tecnología de gestión de la vida cotidiana de las personas.

Es en el contexto de estas transformaciones que la racionalidad médica comienza, cada vez más, a ocuparse de asuntos que con anterioridad eran considerados normales y de remisión espontánea. Para Wakefield et al., este proceso implicaba el riesgo de la multiplicación de los falsos positivos en el diagnóstico de la depresión. Sin embargo, en concomitancia con la medicalización de la tristeza, se 
desarrolló una despatologización de la depresión. Por lo mismo, para Kendler et al., ya no era urgente distinguir entre lo normal y lo patológico, reduciendo la depresión a una experiencia subjetiva de malestar, frente a lo cual el peligro a evitar era el de los falsos negativos.

Desde el punto de vista de la salud colectiva, resulta fundamental comprender que las definiciones de salud y enfermedad no se explican cabalmente a partir del saber científico, ya que ellas, a su vez, expresan relaciones de poder en determinados contextos históricos y sociales ${ }^{(34)}$. Consecuentemente, el presente artículo ha puesto en evidencia que la redefinición del marco epistemológico que registró la psiquiatría contemporánea, da cuenta de una disputa donde "saber" y "poder" están intrínsecamente ligados ${ }^{(59)}$. Si los argumentos de Wakefield et al. fueron de hecho ineficaces, esto se debió, en buena medida, no tanto a su poca capacidad de persuasión científica, sino a una pérdida relativa de poder al interior de la disciplina psiquiátrica. De hecho, la frustración y la sorpresa que sentía Wakefield porque sus argumentos no eran escuchados lo llevan a señalar que la discusión habría dejado de ser científica, puesto que sus oponentes parecían estar totalmente convencidos de su posición y, por ende, cerrados a cualquier crítica $^{(15)}$. Ahora bien, es posible que Wakefield no estuviera del todo equivocado y que la obtusa resistencia que experimentaba fuese el resultado de un juego de poder al interior de la ciencia psiquiátrica, el cual lo enfrentaba, no tanto a su falta de claridad científica, sino a la obsolescencia de la matriz epistemológica que sustenta sus investigaciones y al comienzo de la hegemonía de un nuevo modo de concebir la práctica psiquiátrica, su objeto y sus fines.

\section{REFERENCIAS BIBLIOGRÁFICAS}

1. Ehrenberg A. La fatiga de ser uno mismo: Depresión y sociedad. Buenos Aires: Nueva visión; 1998.

2. American Psychiatric Association. Diagnostic and Statistical Manual of Mental Disorder. Third Edition (DSM-III). Washington DC: American Psychiatric Association; 1980.

3. Shorter E. Before Prozac: The troubled history of mood disorders in psychiatry. Oxford: Oxford University Press; 2009.

4. Wakefield JC, Schmitz MF. When does depression become a disorder?: Using recurrence rates to evaluate the validity of proposed changes in major depression diagnostic thresholds. World Psychiatry. 2013;12(1):44-52. doi: 10.1002/wps.20015.

5. Wakefield JC, Horwitz AV. Psychiatry's continuing expansion of depressive disorder. En: Wakefield JC, Demazeux S, (eds.). Sadness or Depression?: International perspectives on the Depression Epidemic and its Meaning. Dordrecht: Springer; 2016.

6. World Health Organization (WHO). Depression and other common mental disorders: Global health estimates [Internet]. 2017 [citado 13 may 2019]. Disponible en: https://tinyurl.com/ y57565pg.

7. Jirón M, Machado M, Ruiz I. Consumo de antidepresivos en Chile entre 1992 y 2004. Revista 
Médica de Chile. 2008;136(9):1147-1154. doi: 10.4067/S0034-98872008000900009.

8. Gualano MR, Bert F, Mannocci A, La Torre G, Zeppegno P, Siliquini R. Consumption of antidepressants in Italy: Recent trends and their significance for public health. Psychiatric Services. 2014;65(10):1226-1231. doi: 10.1176/appi. ps. 201300510 .

9. Organization for Economic Co-operation and Development. Health at a Glance 2017: OECD Indicators [Internet]. 2017 [citado 10 jul 2019]. Disponible en: https://tinyurl.com/yyfhyng8.

10. World Federation for Mental Health (WFMH). Depression: a global crisis [Internet]. 2012 [citado 13 may 2019]. Disponible en: https://tinyurl.com/ y2uh9ltp.

11. Hidaka BH. Depression as a disease of modernity: Explanations for increasing prevalence. Journal of Affective Disorders. 2012;140(3):205-214. doi: 10.1016/j.jad.2011.12.036.

12. Wakefield JC, Demazeux S (eds.) Sadness or depression?: International perspectives on the depression epidemic and its meaning. Dordrecht: Springer; 2016.

13. American Psychiatric Association. Diagnostic and Statistical Manual of Mental Disorder. Fifth Edition (DSM-5). Washington DC: American Psychiatric Association; 2013.

14. Bandini J. The medicalization of bereavement: (ab)normal grief in the DSM-5. Death Studies. 2015;39(6):347-352. doi: 10.1080/07481187.2014. 951498.

15. Zachar P, First MB, Kendler KS. The bereavement exclusion debate in the DSM-5: A history. Clinical Psychological Science. 2017;5(5):1-17. doi: $10.1177 / 2167702617711284$

16. Kendler KS. Historical precedents for the DSMIII bereavement exclusion criteria for major depression. Psychological Medicine. 2018;48(16):1-10. doi: 10.1017/S0033291718000533.

17. Pies RW. The bereavement exclusion and DSM-5: An update and commentary. Innovations in Clinical Neuroscience. 2014;11(7-8):19-22.

18. American Psychiatric Association. Diagnostic and Statistical Manual of Mental Disorder. Third Edition-Revised (DSM-III-R). Washington DC: American Psychiatric Association; 1987.

19. American Psychiatric Association. Diagnostic and Statistical Manual of Mental Disorder. Forth
Edition (DSM-IV). Washington DC: American Psychiatric Association; 1994.

20. American Psychiatric Association. Diagnostic and Statistical Manual of Mental Disorder. Forth Edition-Revised (DSM-IV-R). Washington DC: American Psychiatric Association; 2000.

21. Zisook S, Shear K, Kendler KS. Validity of the bereavement exclusion criterion for the diagnosis of major depressive episode. World Psychiatric Association. 2007;6(2):102-107.

22. Kendler KS, Myers J, Zisook S. Does bereavement-related major depression differ from major depression associated with other stressful life events? American Journal of Psychiatry. 2008;165(11):14491455. doi: 10.1176/appi.ajp.2008.07111757.

23. Pies RW. Major depression after recent loss is major depression - until proved otherwise. Psychiatric Times [Internet]. 1 dic 2008 [citado 13 may 2019]. Disponible en: https://tinyurl.com/ y4npb89l.

24. Kendler KS, Myers J, Halberstadt LJ. Should the diagnosis of major depression be made independent of or dependent upon the psychosocial context? Psychological Medicine. 2010;40(5):771780. doi: 10.1017/S0033291709990845.

25. Lamb KR, Pies RW, Zisook, S. The Bereavement exclusion for the diagnosis of major depression: to be, or not to be. Psychiatry (Edgemont). 2010;7(7):19-25.

26. Wakefield JC, Schmitz MF, First MB, Horwitz AV. Extending the bereavement exclusion for major depression to other losses: Evidence from the National Comorbidity Survey. Archives of General Psychiatry. 2007;(64)4:433-440.

27. Horwitz AV, Wakefield JC. The loss of sadness: How psychiatry transformed normal sorrow into depressive disorder. Oxford: Oxford University Press; 2007.

28. Wakefield JC. False positives in psychiatric diagnosis: Implications for human freedom. Theoretical Medicine and Bioethics. 2010;31(1):5-17. doi: 10.1007/s11017-010-9132-2.

29. Frances A. Essentials of psychiatric diagnosis. New York: Guilford Press; 2013.

30. Wakefield JC, Horwitz AV, Lorenzo-Luaces L. Uncomplicated depression as normal sadness: Rethinking the boundary between normal and disordered depression. En: DeRubeis RJ, Strunk DR, (eds.). The Oxford Handbook of Mood Disorders. New York: Oxford University Press; 2017. 
31. Sabin JE, Daniels N. Seeking legitimacy for DSM-5: The bereavement exception as an example of failed process. AMA Journal of Ethics. 2017;(19)2:192-198. doi: 10.1001/journalofethic s.2017.19.2.pfor2-1702.

32. Alarcón RD. DSM-5 y la psiquiatría latinoamericana. Revista de Neuro-Psiquiatría. 2013;76(2):6162. doi: 10.20453/rnp.v76i2.1186.

33. Bertolozzi MR, De la Torre Ugarte Guanilo MC. Salud colectiva: fundamentos conceptuales. Salud Areandina. 2012;1(1):24-36. doi: 10.33132/23229659.309.

34. Casallas-Murillo AL. La Medicina Social-Salud Colectiva Latinoamericanas: Una visión integradora frente a la salud publica tradicional. Revista Ciencias de la Salud. 2017;15(3):397-408.

35. Onocko R, Massuda A, Valle I. Salud Colectiva y psicoanálisis: entrecruzando conceptos en busca de políticas públicas potentes. Salud Colectiva. 2009; 4(2):173-185. doi: 10.18294/sc.2008.340.

36. Kawa S, Giordano J. A brief historicity of the Diagnostic and Statistical Manual of Mental Disorders: Issues and implications for the future of psychiatric canon and practice. Philosophy, Ethics, and Humanities in Medicine. 2012;7:2. doi: 10.1186/1747-5341-7-2.

37. Ophir O. Psychosis, psychoanalysis and psychiatry in post war USA: On the borderland of madness. New York: Routledge; 2015.

38. Wilson M. DSM-III and the transformation of American psychiatry: A history. American Journal of Psychiatry. 1993;150(3):399-410.

39. Sandín B. DSM-5: ¿Cambio de paradigma en la clasificación de los trastornos mentales? Revista de Psicopatología y Psicologia Clínica. 2013;18(3):255286. doi: 10.5944/rppc.vol.18.num.3.2013.12925.

40. Shorter E. How everyone became depressed: The rise and fall of the nervous breakdown. New York: Oxford University Press; 2013.

41. American Psychiatric Association. Diagnostic and Statistical Manual of Mental Disorder. First Edition (DSM-I). Washington DC: American Psychiatric Association; 1952.

42. American Psychiatric Association. Diagnostic and Statistical Manual of Mental Disorder. Second Edition (DSM-II). Washington DC: American Psychiatric Association; 1968.

43. Grob GN. The forging of mental health policy in America: World War II to new frontier. Journal of the History of Medicine and Allied Sciences. 1987;(42)4:410-446. doi: 10.1093/jhmas/42.4.410.

44. Mayes R, Horwitz AV. DSM-III and the revolution in the classification of mental illness. Journal of the History of the Behavioral Sciences. 2005;41(3):249-267. doi: 10.1002/jhbs.20103.

45. Kendler KS. Historical precedents for the DSM-III: Bereavement exclusion criteria for major depression. Psychological Medicine. 2018;48(16):2794-2803. doi: 10.1017/S0033291718000533.

46. Freud S. Duelo y Melancolía. En: Sigmund Freud, Obras Completas: Contribución a la historia del movimiento psicoanalítico, trabajos sobre metapsicología y otras obras (1914-1916). Vol. 14. Buenos Aires: Amorrortu; 2008.

47. Clayton P, Desmarais L, Winokur G. A study of normal bereavement. American Journal of Psychiatry. 1968;125(2):168-178.

48. Frances A. Opening pandoras box: The 19 worst suggestions for DSM-5. Psychiatric Times [Internet]. 11 feb 2010 [citado 13 may 2019]. Disponible en: https://tinyurl.com/yyug4r3k.

49. Paris J. The ideology behind DSM-5. En: Paris J, Phillips J, (eds.). Making the DSM-5: Concepts and controversies. New York: Springer; 2013.

50. Muñoz F, Jaramillo LE. DSM-5: ¿Cambios significativos? Revista de la Asociación Española de Neuropsiquiatría. 2015;35(125):111-121. doi: 10.4321/ S0211-57352015000100008.

51. Rose NS. Molecular biopolitics, Somatic ethics and the spirit of biocapital. Social Theory \& Health. 2007;5:3-29. doi: 10.1057/palgrave.sth.8700084.

52. Rose NS, Abi-Rached JM. Neuro: The new brain sciences and the management of the mind. Princeton NJ: Princeton University Press; 2013.

53. Wakefield JC. The DSM-5 debate over the bereavement exclusion: Psychiatric diagnosis and the future of empirically supported treatment. Clinical Psychology Review. 2013;33(7):825-845. doi: 10.1016/j.cpr.2013.03.007.

54. Pies RW, Zisook S. Drs Pies and Zisook respond. En: Wakefield JC, Horwitz AV. Depressive symptoms after loss. Psychiatric Times [Internet]. 13 may 2009 [citado 13 may 2019]. Disponible en: https://tinyurl.com/yymxvqrq.

55. Wakefield JC, Horwitz AV. Depressive symptoms after loss. Psychiatric Times [Internet]. 13 may 2009 [citado 13 may 2019]. Disponible en: https://tinyurl.com/yymxvqrq. 
56. Wakefield JC, Demazeux S. Introduction: depression, one and many. En: Wakefield JC, Demazeux S, (eds.). Sadness or depression?: International perspectives on the depression epidemic and its meaning. Dordrecht: Springer; 2016.

57. Rose NS. Politics of life itself: Biomedicine, power, and subjectivity in the twenty-first century. Princeton: Princeton University Press; 2007.
58. Kass L. Beyond therapy: Biotechnology and the pursuit of happiness. New York: Dana Press; 2003.

59. Foucault M. El poder psiquiátrico: Curso en el Collège de France, (1973-1974). México: Fondo de Cultura Económica; 2005.

\section{FORMA DE CITAR}

Pizarro Obaid F, De La Fabián Albagli R. Un análisis crítico de los debates acerca del duelo y el trastorno depresivo

en la era del Manual Diagnóstico y Estadístico de los Trastornos Mentales. Salud Colectiva. 2019;15:e2319. doi: 10.18294/sc.2019.2319.

Recibido: 16 de mayo de 2019 | Versión final: 19 de julio de 2019 | Aprobado: 27 de septiembre de 2019

Esta obra está bajo una licencia de Creative Commons Reconocimiento-NoComercial 4.0

Internacional. Reconocimiento - Permite copiar, distribuir y comunicar públicamente la obra.

A cambio, se debe reconocer y citar al autor original. No Comercial — Esta obra no puede ser

utilizada con finalidades comerciales, a menos que se obtenga el permiso. 